\title{
Características de liderazgo del administrador educativo de las Instituciones de Educación Superior en México
}

Managerial characteristics of the educational administrators in Higher Education Institutions in Mexico

\section{Volumen 17, Número 3 \\ Setiembre-Diciembre}

pp. 1-26

\section{Este número se publica el $1^{\circ}$ de setiembre de 2017 \\ DOI: http://dx.doi.org/10.15517/aie.v17i3.29871}

\author{
María Teresa de la Garza Carranza \\ Alfonso Cervantes Maldonado \\ Eugenio Guzmán Soria \\ Cecilia Ramos Estrada
}

Revista indizada en REDALYC, SCIELO

Revista distribuida en las bases de datos:

LATINDEX, DOAJ, REDIB, IRESIE, CLASE, DIALNET, SHERPA/ROMEO, QUALIS-CAPES, MIAR

Revista registrada en los directorios:

ULRICH'S, REDIE, RINACE, OEI, MAESTROTECA, PREAL, CLACSO 


\title{
Características de liderazgo del administrador educativo de las Instituciones de Educación Superior en México
}

Managerial characteristics of the educational administrators in Higher Education Institutions in Mexico

\author{
María Teresa de la Garza Carranza ${ }^{1}$ \\ Alfonso Cervantes Maldonado 2 \\ Eugenio Guzmán Soria ${ }^{3}$ \\ Cecilia Ramos Estrada ${ }^{4}$
}

\begin{abstract}
Resumen: La presencia de administradores eficientes en las organizaciones educativas se ha relacionado con los resultados organizacionales y el cumplimiento de metas, puesto que dichos funcionarios son responsables de llevar a cabo las políticas de calidad y para ello deben recurrir a su capacidad de liderazgo. El presente artículo surge de la necesidad de identificar las principales características personales e interpersonales que intervienen en el liderazgo de personas administradoras educativas en las universidades públicas y privadas. El estudio se realizó con base en una muestra de personas administradoras de la COEPES (Comisión Estatal para la Planeación de la Educación Superior) del Estado de Guanajuato en México durante los años de 2015 a 2016. Se desarrolló un modelo de ecuaciones estructurales a través de la técnica de mínimos cuadrados parciales, los resultados demuestran que el modelo es confiable y válido. También se estableció la relación entre las variables capacidades personales e interpersonales con la variable de competencia de liderazgo. Se concluye que las capacidades personales e interpersonales influyen en el liderazgo, tal como lo sugiere la literatura consultada. Esto cobra mayor importancia si se considera que diversos autores especializados en el área, subrayan que el liderazgo es un factor crítico para que las instituciones de Educación superior puedan cumplir con sus objetivos. Aunado a lo anterior, se espera que los resultados de esta investigación contribuyan a que quienes administran las instituciones de educación superior identifiquen sus propias características personales e interpersonales, las cuales influyen en sus habilidades de liderazgo y las fortalezcan, de manera que la gestión estratégica de este tipo de instituciones sea mejorada.
\end{abstract}

Palabras clave: instituciones de enseñanza superior, características directivas, liderazgo.

\begin{abstract}
The presence of efficient administrators in educational organizations has been linked to organizational results and the achievement of goals since these officials are responsible for carrying out quality policies and for this they must resort to their leadership capacity. The present research article arises from the need to identify the main personal and interpersonal characteristics that take part in the leadership of educational administrators in public and private universities. The study was carried out based on a sample of managers of the COEPES (State Commission for the Planning of Higher Education) of the State of Guanajuato in Mexico during the years of 2015 to 2016. A model of structural equations was developed through Partial least squares technique, the results demonstrate that the model is reliable and valid. It also establishes the relationship between the variables personal and interpersonal capacities with the variable of leadership competence. It is concluded that personal and interpersonal skills influence leadership as suggested in the literature. This becomes even more important considering that a number of specialists in the field emphasize that leadership is a critical factor in enabling higher education institutions to achieve their goals. In addition, it is hoped that the results of this research will help managers in higher education institutions identify their own personal and interpersonal characteristics that influence their leadership skills and strengthen them, so that the strategic management of this type of institutions is improved
\end{abstract}

Key words: higher education institutions, managerial characteristics, leadership.

${ }_{1}$ Instituto Tecnológico de Celaya y de la Universidad de la Salle Bajío, México. Dirección electrónica: teresa.garza@itcelaya.edu.mx

2 Universidad Nacional Autónoma de México y de la Universidad de la Salle Bajío, México. Dirección electrónica: acervantes@gmail.com

${ }^{3}$ Instituto Tecnológico de Celaya, México. Dirección electrónica: Eugenio.guzman@itcelaya.edu.mx

${ }^{4}$ Universidad de Guanajuato, México.Dirección electrónica: ceciliagto@gmail.com

Artículo recibido: 10 de octubre, 2016

Enviado a corrección: 08 de mayo, 2017

Aprobado: 3 de iulio, 2017 


\section{Introducción}

Existe una relación entre las estrategias administrativas y la eficiencia universitaria (Friedman, 2003; Pounder 2001 y Spendlove 2007). Los trabajos de investigación sobre el tema sugieren que se debe mejorar el liderazgo de los administradores de la educación superior para cumplir con los estándares demandados. Para desarrollar y operar las estrategias organizacionales se requiere de un administrador-educativo y líderes dentro de las distintas esferas del quehacer universitario. Como en cualquier otra profesión, las habilidades que debe de poseer son diversas ya que, especialmente, tiene el reto de desarrollar integralmente a estudiantes a través de programas académicos, los cuales permitan a los jóvenes mejorar sus oportunidades en la vida.

De acuerdo con el Instituto Mexicano de la Juventud, dos de los principales problemas que enfrentan los jóvenes en la actualidad son conseguir un buen trabajo y tener un buen desarrollo profesional (Instituto Mexicano de la Juventud, 2012). Estos desafíos están relacionados, en parte, con las competencias que pueda adquirir el individuo a través de la educación formal o informal. La educación formal se da a través de los diferentes niveles educativos desde el nivel de primaria hasta el nivel terciario o universitario.

Otro reto importante del administrador-educativo es elevar la calidad de los programas. Como en otras partes del mundo, en México, existen universidades públicas y privadas que contribuyen a la formación especializada de los jóvenes, ofrecen desde el nivel de técnico superior universitario, hasta licenciatura y posgrado. Los dos tipos de universidades (públicas y privadas) están reguladas por la Secretaría de Educación Pública (SEP) de diferente manera. Las universidades privadas deben registrar sus programas ante la SEP a través de un Registro de Validez Oficial (RVOE) para poder operar y ofrecer grados académicos, mientras que las universidades públicas no deben realizar este registro, pero deben de tener mecanismos a su interior que les permitan la apertura de programas educativos y asegurar su calidad. En el caso de las universidades públicas estatales tienen un documento general que rige su actuación. Este documento es denominado Estatuto Orgánico y norma, a través de diversas figuras académicas, la creación de nuevos programas de estudio. Generalmente, una vez que el proyecto de un nuevo programa es realizado se presenta ante una Asamblea General donde el programa es aceptado o rechazado 5 . Otras universidades públicas, como el Tecnológico Nacional de México (TNM), que tienen formas de gobierno centralizadas dado

5 Consultar por ejemplo el Estatuto Orgánico de la Universidad de Querétaro (http://www.uaq.mx/leyes/documentos/EstatutoOrganicoUAQ.pdf) o el de la Universidad de Guanajuato (http://www.ugto.mx/images/pdf/normatividad/estatuto-organico-universidad-guanajuato.pdf) 
que operan a nivel nacional, realizan consultas con pares académicos a través de la dirección general del área.

Las universidades mexicanas, actualmente, se encuentran bajo el escrutinio de diferentes actores, tanto del gobierno federal a través de la SEP como de algunos organismos de la sociedad civil como por ejemplo la Federación de Instituciones Mexicanas Particulares de Educación Superior (Fimpes). Existen también organismos que evalúan y dan acreditaciones a la educación superior. En México, primeramente, se crearon los Comités Interinstitucionales para la Evaluación de la Educación Superior (Ciees) cuyo objetivo es obtener una evaluación diagnostica de los programas educativos y posteriormente, se creó el Consejo para la Acreditación de la Educación Superior (Copaes), que reconoce organismos que están facultados para la acreditación de programas educativos. Estos organismos se especializan en diversas áreas del conocimiento (ingeniería, medicina, administración, diseño, etc.), en la actualidad son 25 y están representados por expertos de cada una de las áreas del conocimiento.

Asimismo, otra estrategia que ha utilizado la SEP para elevar el nivel académico de las universidades públicas es el desarrollo de Perfiles Deseables, así como el de Cuerpos Académicos gestionado por el Programa para el Desarrollo Profesional Docente (Prodep). A nivel de posgrado y de investigación se encuentra el Sistema Nacional de Investigadores que otorga estímulos al profesor-investigador que genera conocimiento científico, y la acreditación de los Programas Nacionales de calidad desarrollados por el Consejo Nacional de Ciencia y Tecnología (Conacyt), este tipo de reconocimientos pueden ser solicitados por universidades públicas y privadas.

Los gobiernos en el mundo, han realizado esfuerzos considerables por elevar los niveles de calidad educativa. De acuerdo con De la Garza Aguilar (2008), existe una tendencia internacional a dar énfasis en la calidad educativa pues la equidad significa ofrecer oportunidades para todos. La estrategia de los gobiernos es mejorar la calidad educativa para la población de diferentes estratos sociales con el objetivo de ofrecer mejores oportunidades de desarrollo del joven. Sin embargo, estos esfuerzos, que involucran gran cantidad de recursos del erario público, pueden ser en vano si no se cuenta con administradores eficaces que lleven a cabo las políticas que se demandan para lograr mejores niveles de calidad educativa. 


\section{Referentes teóricos}

\subsection{Antecedentes del liderazgo en las organizaciones}

Cuando nos referimos a liderazgo se contextualiza a la persona que de alguna manera está a cargo de una organización o de un grupo de personas. En el caso de las instituciones educativas, lo podemos encontrar en la figura del rector, director, jefe de departamento, jefe de desarrollo académico y director de facultad. Para entender la labor de liderazgo dentro del ámbito del administrador-educativo, primeramente, el trabajo contextualizará al líder de manera general para después entender las funciones específicas de liderazgo de la persona que funge como administrador-educativo.

Existen múltiples definiciones y teorías que abordan el tema de liderazgo, algunas hacen énfasis en las características personales del líder, otras a sus funciones dentro de una organización, mientras que algunas más, se centran en las circunstancias particulares del individuo considerado líder, y en algunas otras se apunta a las circunstancias de su entorno. De acuerdo con Black (2015), estos modelos han ido cambiando desde la posición de comando y control del siglo XIX, donde se apreciaba la influencia de la administración científica, hasta modelos más recientes, como los de comportamiento que emergieron en los años cincuenta, y finalmente los modelos de liderazgo transformacional que han sido el paradigma dominante de los últimos 20 años (Bass y Stogdill, 1990).

De acuerdo con Northouse (2013), los enfoques más utilizados para abordar el liderazgo son: el estilo de los rasgos (Stogdill, 1974), el enfoque de estilo (Blake y Mouton, 1985), el enfoque situacional (Hersey y Blanchard, 1993), la teoría de la contingencia (Fiedler y García, 1987), la teoría del intercambio miembro-líder (Graen y Uhl-Bien, 1995) y el liderazgo transformacional (Bass, 1985). Estos enfoques o teorías han sido estudiados ampliamente desde la mitad del siglo XX hasta la fecha por diversos autores y se han aplicado en diversos tipos de organizaciones como la milicia, la educación, las empresas y el gobierno. El objetivo del estudio del liderazgo es identificar un estilo adecuado para el tipo de organización, los seguidores y la personalidad del ejecutivo a cargo; es decir, no existe un modelo que sea universalmente utilizado en toda circunstancia, aunado a ello, se vislumbra que se seguirán desarrollando modelos. Uno de los más recientemente elaborados es el liderazgo de servicio (Greenleaf y Spears, 2002).

Dinh et al. (2014) realizaron un estudio de las teorías de liderazgo tomando en consideración los artículos publicados entre 2000 y 2010 en las 10 publicaciones periódicas más sobresalientes y encontraron que las teorías más citadas en la literatura son el liderazgo 
transformacional, el liderazgo carismático y el liderazgo transaccional. Los representantes más sobresalientes de estas teorías de liderazgo son Antonakis, Avolio y Sivasubramaniam (2003), Judge y Piccolo (2004) y Mumford, Antes, Caughron y Friedrich (2008). El estudio de Dinh et al. (2014) clasifica las teorías de liderazgo en 23 categorías, esto ejemplifica el diverso contexto del tema. Aunque existen múltiples definiciones y posturas alrededor del concepto de liderazgo, Bass (1997) conjuntó las concepciones más comunes relacionadas con este tema, de acuerdo con la siguiente definición:

Los auténticos líderes transformacionales motivan a los seguidores a trabajar en metas trascendentales donde los resultados van más allá de los intereses propios. Los líderes transformacionales mueven a sus seguidores a trascender a sus intereses propios por el bien del grupo, de la organización o el país. (p. 133)

Como se expuso anteriormente, existen diversos factores que influyen en el liderazgo de acuerdo con la teoría en que se analizan, no existe realmente un consenso por parte de los expertos sobre el tema de cuál es el mejor enfoque a utilizar en una determinada situación. Es decir, en algún momento específico un líder podría tomar una actitud transformacional o, bien, utilizar el enfoque situacional de acuerdo con la circunstancia que se presente. En palabras de Northouse (2013):

Después de décadas de la disonancia, los estudiosos de liderazgo están de acuerdo en que: no pueden llegar a una definición común para el liderazgo. Continúa el debate sobre si el liderazgo y la gestión son procesos separados, mientras que otros hacen hincapié en el carácter, habilidad, o aspectos relacionales de liderazgo. Debido a factores tales como la creciente influencia global y las diferencias generacionales, el liderazgo continuará teniendo diferentes significados para diferentes personas. La conclusión es que el liderazgo es un concepto complejo para el que una definición determinada puede ser un largo proceso de cambio. (p. 04)

De lo anterior se desprende que, no hay una teoría de liderazgo universal que pueda ser válida para todas las situaciones bajo las cuales se ejerce este fenómeno, pues diversos cuestionamientos pueden surgir a partir de la revisión de las teorías y sus efectos en la efectividad de la organización. Debido a ello, la teoría del liderazgo sigue evolucionando continuamente. Sin embargo, existen estudios que coadyuvan al fenómeno de manera específica, lo cual ha proporcionado un avance significativo en el entendimiento del 
fenómeno. Por ejemplo, en el caso de las organizaciones latinoamericanas, autores como Castaño et al. (2015) investigaron el liderazgo carismático, la disposición de trabajo en equipo y la autonomía del directivo en 1886 administradores medios de Argentina, Bolivia, Brasil, Colombia, Costa Rica, Ecuador, El Salvador, Guatemala, México y Venezuela. Es decir, de acuerdo con Day, Fleenor, Atwater, Sturm y Mckee (2014) se necesitan estudios específicos que nos ayuden a entender el fenómeno de liderazgo en diferentes ambientes, de ahí que el objetivo del presente estudio sea entender el fenómeno de liderazgo del administrador-educativo en instituciones de educación superior, con el objetivo de mejorar su eficiencia.

\subsection{El liderazgo del administrador-educativo}

El liderazgo académico se entiende tradicionalmente de dos maneras: algunos investigadores lo definen como una serie de tareas y funciones desempeñadas por personas que se encuentran en los diferentes puestos directivos dentro de las instituciones educativas de educación superior, tales como los vicerrectores, directores y jefes de departamento, como lo describe McArthur (2002). Por otro lado, el término liderazgo académico también se utiliza para describir las características o cualidades de profesores que son reconocidos por sus compañeros o estudiantes como líderes en su especialidad.

En consecuencia, el liderazgo en el ámbito académico se entiende, primeramente, ya sea como la función de un cargo directivo en particular dentro de una institución educativa, lo cual es independiente de las características de la persona que lo ocupa. O bien, como los rasgos de una persona con un desempeño académico sobresaliente, que es difícil de imitar, aprender o desarrollar. López Yáñez y Sánchez Moreno (2009) identifican claramente estos dos criterios, ellos proponen, para los líderes administrativos, las funciones de bienestar de la organización, los sistemas de financiamiento y generación de recursos, así como la construcción de un clima saludable dentro de las Instituciones de Educación Superior (IES); mientras que, las funciones de los líderes académicos se centran en las directrices académicas, el balance y la administración del currículo, funciones de investigación, entre otras.

En este artículo consideraremos el término de liderazgo del administrador-educativo como la función que ocupa una persona en un puesto directivo en una IES, se tomarán en consideración desde los niveles más altos, como el de rectores o directores, hasta los niveles operativos, como los que ocupan los jefes de departamento. A continuación, describiremos 
algunos enfoques sobre los cuales ha sido estudiada la función del líder académico, ya que existe una relación entre la calidad del liderazgo ejercido y los resultados de la escuela y del estudiantado (Bush, 2007). Cabe señalar que el tema no es exclusivo de las instituciones de educación superior debido a que este tipo de personal se desarrolla en cualquier nivel educativo.

De acuerdo con Jones, Lafoe, Harvey y Rayland (2012) el liderazgo adecuado para la administración de las IES es el que reconoce la compleja interacción entre los sujetos, objetos e instrumentos, normas, la comunidad y la división del trabajo para desarrollar la capacidad de liderazgo. Un estudio ampliamente citado en la literatura, sobre el liderazgo del administrador-educativo, es el de Ramsden (1998), quien realizó un estudio en jefes de departamentos académicos en universidades de Hong Kong, Nueva Zelanda, Inglaterra, Singapur y Australia. El estudio consistió en entrevistar a administradores educativos, a ellos se les pedía que hicieran una reseña sobre los líderes que, según su consideración, hubieran hecho un trabajo excepcional en proveer un trabajo académico altamente efectivo. Las respuestas fueron las siguientes:

1) Tienen una visión que está en armonía con el equipo académico (incluyendo la habilidad de ver más allá de los problemas inmediatos), poseen una integridad académica (autenticidad, digno de confianza, respetuoso, justo, enfático, liderazgo con ejemplo) y son eficientes.

2) Son capaces de fusionar un grupo diverso, en una organización coherente con un ideal claro y común.

3) Son buenos con las relaciones y capaces de actividades políticas en su organización, así como de una alineación estratégica (emprendedores, toman riesgos, con visión de futuro).

4) Inspiran (confianza), escuchan, son capaces de sobrevivir a reveces, dan confianza de a dónde van, proveen esperanza, trabajan colaborativamente y dan seguimiento a sus decisiones.

5) Proveen de reconocimiento sólido en la administración del desempeño (vinculan metas individuales a las departamentales); apoyan el aprendizaje del staff (profesores y personal administrativo). Buscan oportunidades para los profesores jóvenes, organizan las recompensas y establecen un entorno donde se reconoce el buen trabajo. 
El estudio de Ramsden ha sido citado ampliamente en la literatura (Bryman, 2007 y Becher y Trowler, 2001) ya que fue uno de los primeros en describir la labor del líder académico. Otro estudio ampliamente citado en la literatura es el de Spendlove (2007), el cual consiste en entrevistas semiestructuradas con líderes académicos administrativos acerca de las competencias efectivas. Los hallazgos se agruparon en tres categorías de acuerdo con lo siguiente:

1) Actitud (lo que los buenos líderes son): Conscientes de sí mismos, flexibles, abiertos, honestos, discretos, visibles, sensitivos, dispuestos a cometer errores, aceptan consejos y apoyo.

2) Conocimientos (lo que los buenos líderes saben): Entienden cómo trabaja el sistema universitario, entienden el proceso académico.

3) Comportamiento o mixtos (lo que los buenos líderes hacen): Trabajan para mantener la credibilidad académica y buscan que sea respetada, actúan como un modelo, piensan ampliamente y de forma estratégica, se relacionan con otros, saben escuchar, negocian, se comunican claramente, delegan, motivan, actúan como mentores, construyen equipos.

Berdrow (2010), por su parte, elaboró un modelo en el que se identifican seis categorías principales de actividades de un directivo de una IES, de las cuales cuatro son de carácter administrativo y las otras se relacionan con el liderazgo. Las categorías son: desarrollo estudiantil, desarrollo docente, comunicación, administración de operaciones, innovación y mejora del clima organizacional, como se muestra en la figura 1. 
Figura 1

Rol del administrador-educativo

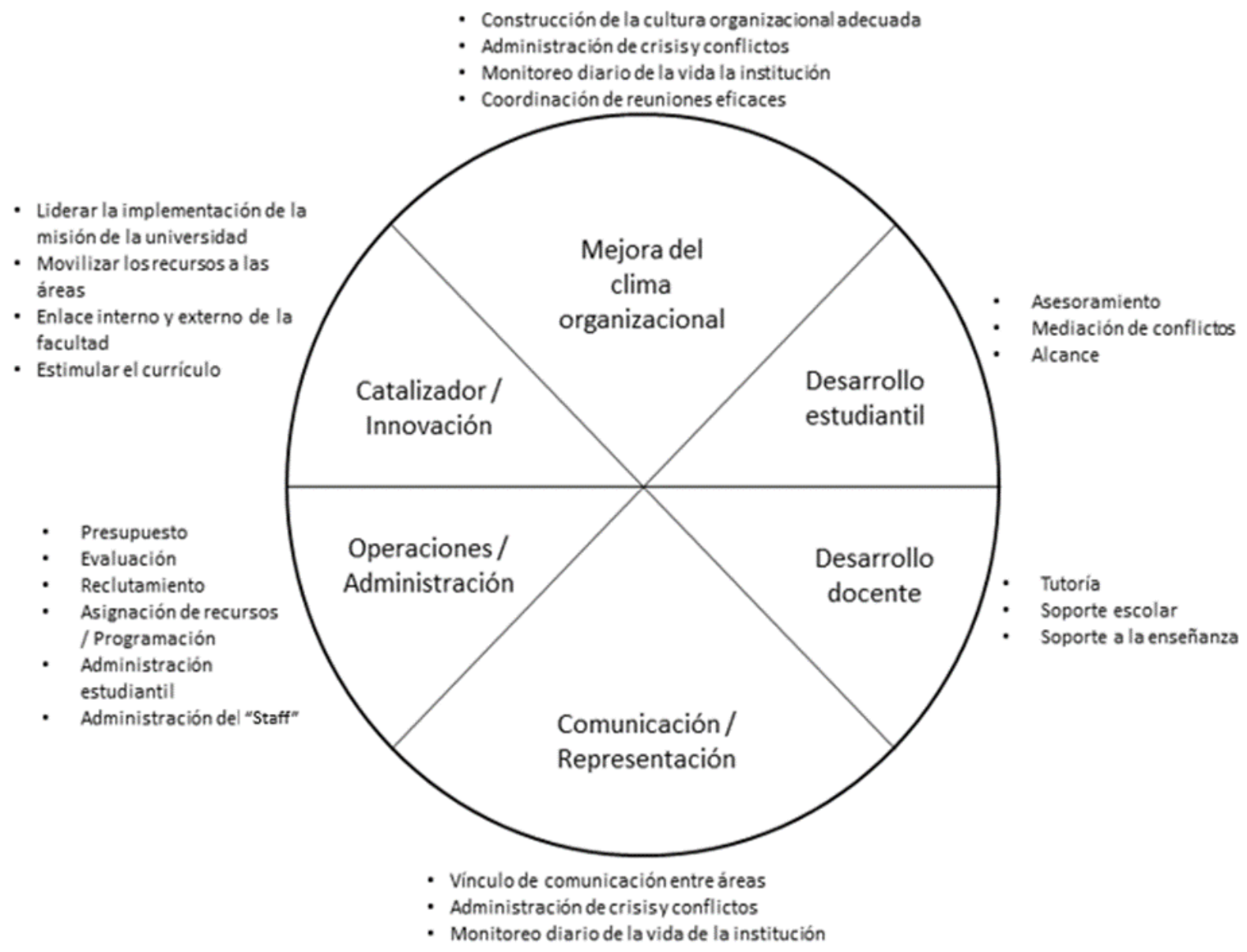

Fuente: Adaptado a partir de Berdrow (2010).

Como se puede observar en la figura 1, el rol de un directivo educativo no es fácil, tiene que cumplir diversas actividades que, por su complejidad, requieren de habilidades especiales o competencias. Se debe ser un líder que abarque diversas funciones y tareas administrativas (operaciones/administración), esté en contacto con diferentes actores sociales (comunicación/representación), lleve a alcanzar niveles de excelencia en el ámbito académico (desarrollo docente y estudiantil), busque las mejores prácticas académicas y organizacionales (catalizador/innovador) y conduzca a toda la organización a mejores niveles de desempeño (mejora del clima organizacional). 
Otro estudio importante que aporta a la comprensión de la función del directivo de las IES es el que realizaron Scott, Coates y Anderson (2008) y consistió en entrevistas a 513 administradores educativos de 20 universidades australianas, tomaron como ejes las capacidades y competencias necesarias para realizar de forma efectiva esta función. Su trabajo comprendió diversas posiciones, desde las más altas (miembros de las juntas de consejo, rectores y directivos) hasta personal operativo como jefes de departamento, coordinadores de programa y jefes de departamento de servicios (servicios escolares, asuntos estudiantiles). Los investigadores, encontraron un modelo de capacidades y competencias en tres ejes principales:

1) Capacidades personales e interpersonales. Se describe la dimensión humana del modelo y se refiere a la capacidad de manejar las emociones para tolerar la ambigüedad, mantener la calma y no sobre reaccionar ante situaciones difíciles; es la inteligencia emocional (Goleman, 1998) que se debe de manejar en cualquier situación que conlleve trabajo de relaciones humanas.

2) Capacidades cognitivas. Se refiere a poder realizar diagnósticos adecuados de situaciones que conlleva la administración educativa dentro del ámbito universitario.

3) Competencias de liderazgo. Estas comprenden las habilidades de proveer un andamio para dar forma a la respuesta correcta y entregarla de manera conjunta a todos los actores implicados.

En el modelo de Scott et al. (2008), existe una diferencia entre competencias y capacidades. La competencia que puede adquirir una persona, se refiere a "alguien que posee las habilidades clave y el conocimiento requerido para entregar las tareas que conforman un trabajo específico o son necesarios para ejecutar una operación particular de manera efectiva" (p. 10), mientras que la capacidad se refiere al "nivel de talento o don requerido para producir resultados y ofrecer innovaciones bajo pruebas, incertidumbre y cambiando constantemente las situaciones humanas y técnicas" ( $p$. 11). Bajo esta perspectiva las competencias pueden ser fragmentadas en listas, mientras que las capacidades son más holísticas, integradoras, multidimensionales y creativas.

La Organización de las Naciones Unidas para la Educación, la Ciencia y la Cultura (Unesco, 2000) en el documento "Desafíos de la Educación", menciona seis prioridades estratégicas que se deben de afrontar para dar respuesta a los retos de un mundo cambiante que demanda mejor educación. Los desafíos son: 
1) Invertir en la formación de recursos humanos. Se deben generar e invertir recursos suficientes para formar profesores, investigadores y cuerpos administrativos que constituyen la universidad.

2) Multiplicar las instancias de encuentro e intercambio horizontal dentro del sistema educativo. Esto se refiere a compartir experiencias entre universidades y aun dentro de las mismas facultades para encontrar las mejores prácticas que lleven a resultados de éxito.

3) Reinstaurar los liderazgos. Es necesario que existan líderes que guíen el trabajo en equipo y que lleven a esfuerzos colaborativos entre los diferentes cuerpos académicos que conduzcan a resultados tangibles dentro de las IES.

4) Ampliar la capacidad de decisión a nivel local. Para que los sistemas educativos de nivel superior mejoren su capacidad de transformación local es necesario que se aborden retos del entorno. Los sistemas centralizados donde se privilegia la toma de decisiones poco participativa por parte de los grupos colegiados, pueden presentar deficiencias en los currículos poco adaptados a las necesidades regionales. Este puede ser el caso de los grandes sistemas educativos que se han privilegiado en México (Tecnológico Nacional de México, Universidades Politécnicas, Universidades Tecnológicas) donde la mayoría de las decisiones se llevan a cabo de forma centralizada.

5) Definir múltiples mecanismos y procedimientos de evaluación y generación de responsabilidades institucionales por los resultados. Para lograr la eficiencia en las IES es necesario identificar y evaluar los procesos que llevan a realizar la función integral de educación. Se debe de considerar que uno de los principales retos de las universidades es la formación de profesionales y se deben de integrar procedimientos que aseguren que el estudiantado pueda llevar a buen término su titulación.

6) Apostar a la creación de nuevos modos de articulación del sistema educativo con el entorno. Para garantizar que los esfuerzos de las IES detonen el desarrollo regional y nacional es necesario articular mecanismos de vinculación con diversos sectores productivos, sociales y gubernamentales. En este punto es necesario desarrollar mejores mecanismos de transferencia de tecnología que lleve a las organizaciones a mejores prácticas de innovación y desarrollo tecnológico. 
Como se puede observar, el trabajo del líder académico es complicado y depende de gran cantidad de factores para lograr que su gestión sea exitosa. Especialmente, la gestión en las IES públicas es aún más compleja, ya que es muy difícil penalizar las conductas que no favorecen la productividad pues los derechos creados por los trabajadores y los sindicatos no permiten utilizar algunas estrategias de cambio organizacional (De la Garza Carranza, 2011).

\section{Contextualización del problema}

Para poder llevar a cabo una gestión adecuada de las IES, es necesario profundizar en las capacidades y competencias que debe tener el administrador-educativo. Como se mencionó anteriormente, para alcanzar las metas de la educación superior se requiere de directivos eficientes que hagan las funciones de gestores de recursos y logren que, tanto profesores, estudiantes y programas educativos concurran de manera efectiva y eficiente para abatir los rezagos que se tienen en la educación terciaria. En el caso del Estado de Guanajuato y de acuerdo con el Sistema Nacional de Información Estadística (2016) de la SEP, existen 326 planteles de educación superior, de los cuales 79 son públicos y 247 son privadas. Es necesario mencionar que en los dos últimos periodos del Gobierno del Estado se ha enfatizado en la creación de universidades públicas, pero aún se deben hacer esfuerzos en este sentido. Para contextualizar la situación de la educación superior en el estado de Guanajuato, se presentan datos relevantes sobre absorción en la siguiente tabla.

Tabla 1. Población de la matrícula universitaria, docente y cobertura en Guanajuato y México (Período 2015- 2016)

\begin{tabular}{|l|r|r|r|r|r|r|}
\hline $\begin{array}{l}\text { Educación superior } \\
\text { (Guanajuato) }\end{array}$ & \multicolumn{1}{c}{ Total } & Mujeres & Hombres & Docentes & Escuelas & Cobertura $^{1}$ \\
\hline Licenciatura & 117054 & 58363 & 58691 & 12225 & 326 & $24.3 \%$ \\
\hline Publico & 73680 & 33272 & 40588 & 6270 & 79 & \\
\hline Privado & 53079 & 30248 & 22831 & 8876 & 247 & \\
\hline $\begin{array}{l}\text { Educación superior } \\
\text { (México) }\end{array}$ & 3411326 & 1675886 & 1735442 & 328360 & 4768 & $35.8 \%$ \\
\hline
\end{tabular}

${ }^{1}$ Cobertura de educación superior (pública y privada) sobre la población total mexicana de personas entre 18 a 22 años de edad. No incluye posgrado e incluye la modalidad no escolarizada

Fuente: Elaborado a partir del Sistema Nacional de Información Estadística Educativa de la SEP de México (2016a y 2016b).

Para regular la planeación educativa en cada uno de los estados en México, existe la Comisión Estatal para la Planeación de la Educación Superior (Coepes), esta comisión es una organización no gubernamental que regula la apertura de nuevas carreras, está 
integrada por los diferentes directores o rectores de la educación superior de cada Estado y busca incrementar la calidad y pertinencia de los programas ya sean las instituciones públicas o privadas.

La Coepes del Estado de Guanajuato tiene instituciones públicas y privadas afiliadas que contribuyen de igual manera a la formación de estudiantes a nivel universitario de pregrado y posgrado. Inclusive, este organismo ha ofertado cursos para fortalecer los cuadros académicos y directivos. Este proyecto de investigación pretende ser una fuente de información para la toma de decisiones en la mejora del personal administrativo de las IES del Estado de Guanajuato ya que permitirá identificar aquellos rasgos en los cuales el personal tiene fortalezas y debilidades en relación con las características sugeridas a nivel internacional.

\subsection{Objetivos del estudio}

El objetivo de este proyecto de investigación fue realizar un modelo que explicara las competencias del liderazgo de los administradores-educativos en función de sus capacidades personales e interpersonales. Para ello, se tomó como base los cuestionarios utilizados por Scott et al. (2008). Por tanto, la pregunta de investigación es ¿cómo afectan las capacidades personales e interpersonales de los administradores educativos su competencia de liderazgo?

La hipótesis de trabajo para este proyecto de investigación es: H1. Existen capacidades personales e interpersonales que afectan la competencia del liderazgo del administrador-educativo.

\subsection{Instrumentos de medición}

Los cuestionarios utilizados para probar la hipótesis fueron: 1) Capacidades personales del administrador-educativo 2) Capacidades interpersonales del administrador-educativo y 3) Competencias de liderazgo del administrador-educativo. Estos cuestionarios fueron desarrollados por Scott et al. (2008). Se utilizó una escala Likert de cinco puntos para medir los ítems. Es importante hacer notar que se hizo primeramente una traducción al español y después del español al inglés para utilizar el procedimiento estándar para la aplicación de un cuestionario desarrollado en una lengua no nativa, tal como lo sugieren, en su libro, Palacios y Vargas (2011). Esto aseguró la contextualización del cuestionario al sistema educativo de México. Adicionalmente, ya se había realizado una prueba piloto del cuestionario tomando 
en cuenta a todo el cuerpo de administradores educativos de una institución IES y los resultados fueron aceptables (Sánchez Gómez, De la Garza Carranza y Cervantes Maldonado, 2013).

\section{Metodología y resultados estadísticos}

La metodología del proyecto de investigación fue cuantitativa, transversal, exploratoria y causal. Se dice que un estudio es cuantitativo cuando se recopilan datos numéricos basados en cuestionarios. El presente estudio, como fue aplicado en un momento en el tiempo, se puede decir que es transversal y los resultados son descriptivos o exploratorios; no obstante, al buscar relaciones causa y efecto es también un estudio causal. Se aplicaron cuestionarios a una muestra de administradores educativos de diversas universidades seleccionadas mediante la técnica de muestreo aleatoria, se tomó como universo las universidades asociadas a la Coepes de Guanajuato. Se enviaron cartas firmadas por el Hno. Andrés Govela Gutiérrez director de Coepes, quien invitó a contestar el cuestionario a los directores, rectores o encargados administrativos de las siguientes instituciones:

1) Universidad de Guanajuato.

2) Universidad Politécnica de Guanajuato.

3) Universidad Politécnica de Pénjamo.

4) Sistema Avanzado de Bachillerato y Educación Superior.

5) Unidad Profesional Interdisciplinaria de Ingenierías Campus Guanajuato del Instituto Politécnico Nacional.

6) Instituto Tecnológico Superior de Irapuato.

7) Universidad Pedagógica Nacional Plantel Celaya.

8) Universidad Tecnológica de Salamanca.

9) Instituto Tecnológico de Celaya.

10) Instituto Tecnológico de Monterrey Campus Irapuato.

11) Universidad de Estudios Profesionales de Ciencias y Artes.

12) Universidad Latina de México.

Se recibieron 54 respuestas válidas de los directivos y mandos medios de Instituciones de Educación Superior durante los meses de octubre de 2015 a febrero de 2016. Cabe resaltar que la muestra sugerida por Hair, Hult, Ringle y Sarstedt (2013) para la utilización de mínimos cuadrados parciales tomando en consideración una potencia de la prueba del 80\% 
y un nivel de significancia de $5 \%$ es de 48 , aduciendo que el universo es infinito. La aplicación se llevó a cabo mediante una herramienta electrónica de código abierto llamada limesurvey. Se realizó la invitación a participar en el estudio mediante la Coepes del Estado de Guanajuato, la aplicación fue confidencial por lo que no se identificó la procedencia de las instituciones educativas. El análisis metodológico estadístico se dividió en tres partes. a) Análisis descriptivo de los datos donde se obtuvieron los datos de media y varianza y de correlaciones bivariadas mediante el software SPSS (Versión 21), b) Análisis de confiabilidad y validez de los cuestionarios, mediante el mismo software y c) Análisis de ecuaciones estructurales mediante el software Smart-PLS (Versión 3). El nivel de significancia establecido para probar la hipótesis fue de $\alpha=0.05$.

\subsection{Análisis descriptivo de los datos}

Respecto al género de los participantes, 24 de ellos respondieron pertenecer al sexo masculino, 24 al sexo femenino y 6 no respondieron. Referente a la edad de los participantes, 13 de ellos tiene una edad de 35 años o menos, 26 participantes tienen entre 36 a 45 años, 12 participantes pertenecen al grupo de 46 a 55 años y 3 participantes están en el rango de 56 a 65 años. En este sentido, se denota un equilibrio de género y edades en relación con el cargo ocupado.

El grado académico de los participantes muestra que 7 de ellos tienen grado de licenciatura, 3 nivel de especialidad, 31 grado de maestría y 13 cuentan con grado de doctor. En el puesto o posición jerárquica que desempeñan los participantes se identifican a 18 jefes de departamento, 19 coordinadores de programas académicos, 4 coordinadores de direcciones de apoyo, 2 coordinadores de plantel, 3 directores de unidades académicas, 2 directores de áreas de apoyo, 1 director de campus, 1 director de plantel y 1 secretario académico. La antigüedad en el puesto actual de los participantes se distribuye de la siguiente manera: 16 participantes mencionan tener antigüedad menor de un año, 17 participantes tienen una antigüedad de 1 a 3 años, 11 participantes presentan una antigüedad de 4 a 6 años, 9 de ellos tienen antigüedad de 7 a 10 años y 1 participante tiene una antigüedad mayor a los 10 años. En cuanto a la experiencia laboral de los participantes, 29 de ellos mencionaron tener experiencia en otras instituciones educativas y 18 mencionaron haber tenido experiencia en otro tipo de organizaciones. En general, podemos decir que la mayoría de las personas que contestaron el cuestionario ocupa una posición de 
jefatura de departamento, tiene maestría, con una antigüedad mayor a los 3 años. En la tabla dos se muestran los descriptivos de medias y desviación estándar de los datos.

Tabla 2. Media y desviación estándar de las capacidades personales e interpersonales y competencias de liderazgo

\begin{tabular}{|l|r|r|}
\hline \multicolumn{1}{|c}{ Variable } & Media & $\begin{array}{c}\text { Desviación } \\
\text { Estándar }\end{array}$ \\
\hline Capacidades personales & 4.44 & 0.34 \\
\hline Capacidades interpersonales & 4.43 & 0.47 \\
\hline Competencias de liderazgo & 4.45 & 0.36 \\
\hline
\end{tabular}

Fuente: Elaboración del autor a partir de los datos recopilados para la presente investigación, de los administradores-educativos del Estado de Guanajuato, México, 2016.

Adicionalmente, con el fin de evaluar si existe una relación entre las variables estudiadas, se obtuvo el coeficiente de correlación de Pearson. La tabla 3 muestra los índices de las relaciones bivariadas entre las variables estudiadas. Como se puede observar, las relaciones son positivas y significativas.

Tabla 3. Correlaciones bivariadas de Pearson de las capacidades personales e interpersonales y competencias de liderazgo con su respectiva significancia estadística

\begin{tabular}{|l|r|r|r|}
\hline Capacidades & \multicolumn{1}{c}{$\begin{array}{l}\text { Capacidades } \\
\text { personales }\end{array}$} & \multicolumn{1}{c}{$\begin{array}{l}\text { Capacidades } \\
\text { interpersonales }\end{array}$} & \multicolumn{1}{l}{$\begin{array}{l}\text { Competencia } \\
\text { de liderazgo }\end{array}$} \\
\hline $\begin{array}{l}\text { Capacidades } \\
\text { personales }\end{array}$ & 1 & & \\
\hline $\begin{array}{l}\text { Capacidades } \\
\text { interpersonales }\end{array}$ & $0.607^{* *}$ & & \\
\hline $\begin{array}{l}\text { Capacidades } \\
\text { de liderazgo }\end{array}$ & 0.001 & & \\
\hline
\end{tabular}

** La correlación es significativa al nivel de 0.01

Fuente: Elaboración del autor mediante a partir de los datos recopilados para la presente investigación, de los administradores-educativos del Estado de Guanajuato, México, 2016.

\subsection{Validación de los cuestionarios}

Para la validación de los cuestionarios, se realizaron pruebas de Cronbach y análisis factorial exploratorio mediante el software SPSS. El método de consistencia interna basado en el alfa de Cronbach permite estimar la confiabilidad del cuestionario medida a través de un conjunto de ítems que se espera que midan el mismo constructo o dimensión teórica. Como se muestra en la tabla 3, los coeficientes de alpha de Cronbach mostrados para cada uno de los cuestionarios utilizados van de 0.703 a 0.889 ; los valores deseables para el estadístico deben de ser superiores a 0.7 y son deseables los valores cercanos a 1 de 
acuerdo con Field (2005). Sin embargo, Cortina (1993) hace una observación con respecto a este estadístico y menciona que, si se tienen un mayor número de ítems o preguntas en el cuestionario, el estadístico tenderá a ser mayor. Es debido a esta razón que se deben de aplicar otras pruebas que nos ayuden a entender si el instrumento es confiable.

Tabla 4. Coeficientes de alpha de cronbach pertenecientes a las escalas de medición de capacidades personales e interpersonales y competencias de liderazgo

\begin{tabular}{|l|c|}
\multicolumn{1}{|c|}{ Escala } & \multicolumn{1}{c|}{$\begin{array}{c}\text { Alpha de } \\
\text { cronbach }\end{array}$} \\
\hline $\begin{array}{l}\text { Capacidades } \\
\text { personales }\end{array}$ & 0.703 \\
\hline $\begin{array}{l}\text { Capacidades } \\
\text { interpersonales }\end{array}$ & 0.889 \\
\hline $\begin{array}{l}\text { Capacidades } \\
\text { de liderazgo }\end{array}$ & 0.858 \\
\hline
\end{tabular}

Fuente: Elaboración del autor mediante a partir de los datos recopilados para la presente investigación, de los administradores-educativos del Estado de Guanajuato, México, 2016.

Como segundo paso para evaluar la confiabilidad de la escala, se utilizó el análisis factorial exploratorio. Este análisis nos permite identificar los factores asociados a la escala. Para obtener un cuestionario probado, se requieren tres tipos de pruebas asociadas a este análisis: El indicador de Kaiser Meyer y Olkin (KMO), la prueba de esfericidad de Bartlett y la varianza explicada por los ítems (variables) que constituyen la escala. El test KMO contrasta las correlaciones parciales entre variables, puede ser calculado para variables individuales o múltiples y relaciona el cuadrado de la correlación entre las variables con el cuadrado de la relación parcial entre las variables, de tal manera que es una medición de la adecuación de la muestra, cuanto más cerca se obtenga el valor de 1 es mejor la prueba KMO.

Esto implica que los patrones de correlaciones son relativamente compactos; los valores mayores a 0.60 se consideran aceptables. La prueba de esfericidad de Bartlett evalúa la aplicabilidad del análisis factorial de las variables estudiadas, de manera que al encontrarse un índice inferior a 0.05 se rechaza la hipótesis de que la matriz de correlaciones es una matriz identidad para determinar si las correlaciones son significativas entre las variables y, por último, la varianza explicada nos indica qué tanto el cuestionario explica el fenómeno estudiado. Cuanto mayor sea la varianza explicada por el cuestionario es mejor; por ejemplo, en el cuestionario de las capacidades de liderazgo, el fenómeno estudiado se explica en $72 \%$, esto implica que el $28 \%$ de la varianza se debe a otros factores no explicados por el cuestionario. Los resultados se muestran a continuación en la tabla 5. 
Tabla 5. Estadístico KMO, prueba de Bartlett y varianza explicada pertenecientes a las escalas de medición de capacidades personales e interpersonales y competencias de liderazgo

\begin{tabular}{|l|r|r|r|}
\hline & Kscala & \multicolumn{3}{c}{$\begin{array}{c}\text { Prueba de Bartlett } \\
\text { (significancia de la } \\
\text { prueba) }\end{array}$} & Varianza Explicada \\
\hline dades & 0.604 & $\leq 0.01$ & $64 \%$ \\
\hline ales & 0.826 & $\leq 0.01$ & $72 \%$ \\
\hline idades & 0.70 & $\leq 0.01$ & $68 \%$ \\
\hline tencias de & & &
\end{tabular}

Fuente: Elaboración del autor mediante a partir de los datos recopilados para la presente investigación, de los administradores-educativos del Estado de Guanajuato, México, 2016.

\subsection{Modelo de ecuaciones estructurales}

Una vez que se comprobó la confiabilidad y validez del cuestionario se procedió a desarrollar un modelo de ecuaciones estructurales mediante la técnica de mínimos cuadrados parciales. De acuerdo con Lévy-Mangin y Varela (2006), un mayor número de investigadores adopta metodologías que utilizan los modelos causales o de ecuaciones estructurales para explicar los fenómenos de las ciencias sociales. Estos modelos son superiores a las técnicas estadísticas tradicionales pues permiten incorporar constructos abstractos y no observables como lo son, en este caso, las variables capacidades interpersonales (CI), capacidades personales (CP) y competencias de liderazgo (CL) definidas anteriormente en los cuestionarios utilizados. Los enfoques más conocidos son los basados en el análisis de la estructura de covarianzas que utilizan los softwares como el STATA, AMOS, etc. Sin embargo, Wold (1975) propuso un modelo alternativo con el objetivo de reflejar las condiciones teóricas y empíricas de las ciencias sociales y del comportamiento, en las que son habituales las situaciones con teorías no suficientemente sólidas y escasa información disponible, donde el tamaño de muestra muy a menudo es pequeño. A este enfoque se le conoce como "Ecuaciones Estructurales utilizando PLS"; las siglas PLS significan Partial Least Squares en inglés.

Este método se enfoca en maximizar la varianza de las variables dependientes explicadas por las independientes en vez de reproducir la matriz de covarianza empírica (Valdéz Blanco, 2010). Como en cualquier SEM, un modelo de PLS se compone de una parte estructural que refleja las relaciones entre las variables latentes y un componente de medición que muestra cómo las variables latentes y sus indicadores están relacionados, pero también tiene un tercer componente, las relaciones de carga que se utilizan para estimar las 
relaciones entre las variables (Chin y Newsted, 1999). En cuanto al tamaño de muestra y de acuerdo con los criterios presentados por Hair, et al. (2013), "el número máximo de ítems apuntando a un constructo en este caso es de 6 y de manera teórica tendríamos un nivel de significancia de un rango entre 95 y 99\%”, esto según la tabla explicativa presentada por ellos (p. 21).

El modelo que a continuación se presenta (figura 2), representa la interacción que las variables capacidades personales $(C P)$ y las capacidades interpersonales $(C l)$ tienen con las competencias de liderazgo (CL); y es el resultado de diversas iteraciones a través de la prueba de los diversos ítems de cada una de las tres variables del estudio (capacidades personales, capacidades interpersonales y capacidades de liderazgo). Para lo obtención del modelo no existe un algoritmo específico y es más bien la relación con la teoría la que proporciona la propuesta del modelo. En dicho modelo, las variables circulares representan las variables latentes del estudio, y las variables en los recuadros son los ítems que son los mejores indicadores de cada una de las variables estudiadas. Podemos observar en la figura 2 que tanto la variable latente $\mathrm{Cl}$ y $\mathrm{CP}$ son variables formativas, ya que consideramos que ambas son independientes y la variable CL es una variable reflexiva, pues es consecuencia de las variables CI Y CP (Rossiter, 2002). 
Figura 2

Modelo de ecuaciones estructurales

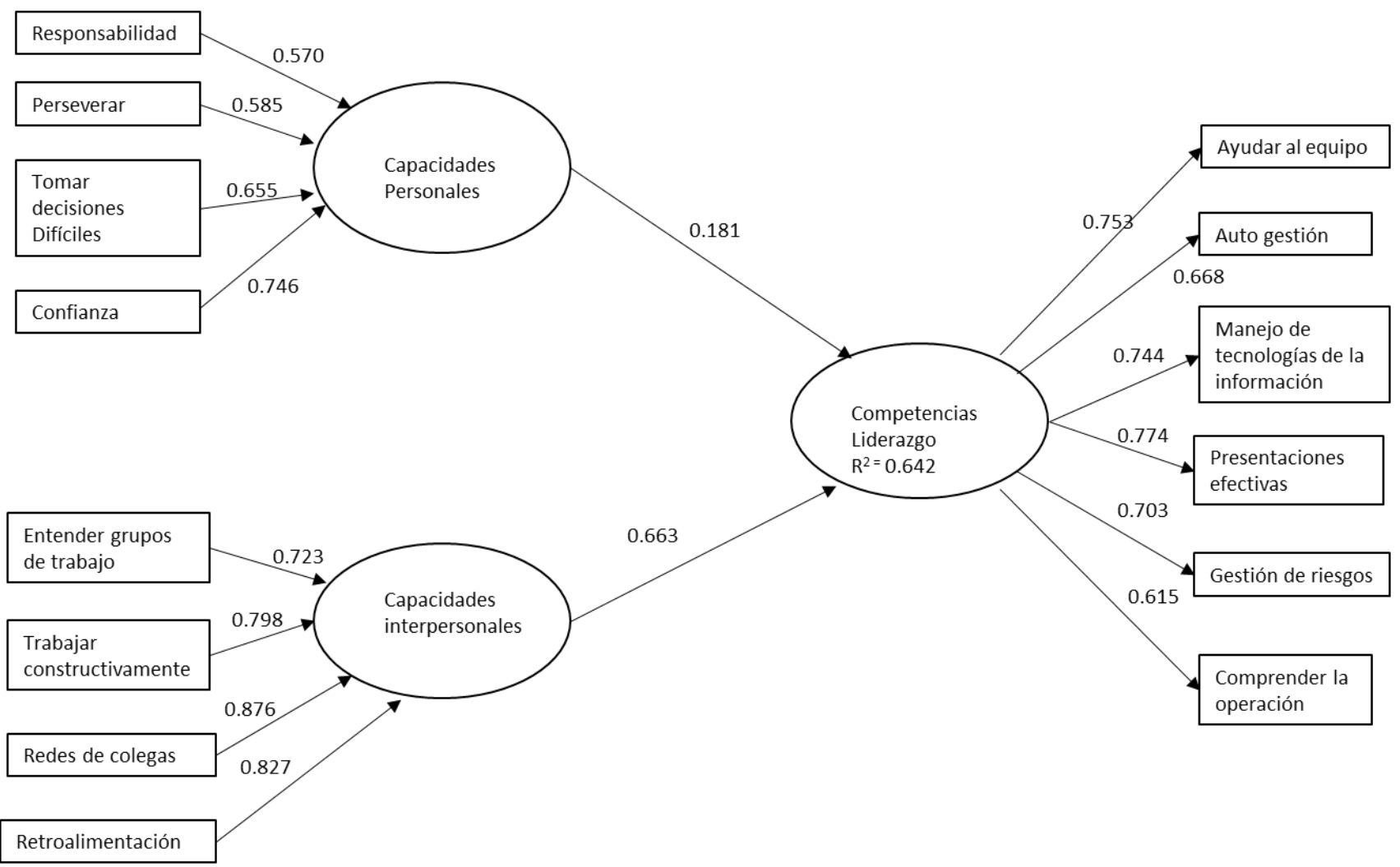

Fuente: Elaboración del autor a partir de los datos recopilados para la presente investigación, de los administradores-educativos del Estado de Guanajuato, México, 2016.

El modelo estudiado, se realizó en el software Smart-PLS desarrollado por Ringle, Wende y Will (2005) debido a su facilidad de manejo, pero existen otros softwares en el mercado que se manejan bajo el mismo concepto como el $R$. Para probar la confiabilidad y validez del modelo existen ciertos indicadores que se deben cumplir (Hair et al., 2013 y Wong 2013). Estos indicadores son diferentes a los que generalmente utilizan los procedimientos mínimos cuadrados ordinarios para construcción de ecuaciones estructurales y que son básicamente el RMSEA (Aproximación al error cuadrado medio), el TLI (Indicador Tucker Lewis) y el CFI (Índice de ajuste comparativo). En este caso donde se aplicaron mínimos cuadrados parciales en la construcción de ecuaciones estructurales, primeramente, se debe comprobar que las cargas externas sean mayores que 0.70 pero un indicador mayor que 0.4 es aceptable para trabajos exploratorios; estos datos se encuentran en las flechas del modelo y muestran la confiabilidad del indicador. En segundo término, se debe verificar que la fiabilidad compuesta o composite reliability sea mayor que 0.70 ; en este caso es de 
0.86 ( $\mathrm{t}=32.38, \mathrm{p} \leq \mathrm{0.001})$, esto demuestra la consistencia interna. El siguiente parámetro a probar se refiere a la validez del modelo y se utiliza la validad de convergencia o AVE que debe de ser igual o mayor de 0.5 (Bagozzi y Yi, 1988); en este caso, el valor obtenido a través del software es de $0.506(t=9.91, p \leq 0.001)$. Finalmente, la correlación al cuadrado del modelo $\left(R^{2}\right)$ es $0.642(t=8.63, p \leq 0.001)$; la $R^{2}$ ajustada es de $0.628(t=7.63, p \leq 0.001)$; con un alpha de cronbach total de $0.803(\mathrm{t}=17.64, \mathrm{p} \leq 0.001)$ que indica un estimado de la confiabilidad basada en las correlaciones de todas las variables observadas. En conclusión, es posible decir que el modelo es confiable y válido.

\section{$5 \quad$ Discusión de resultados y conclusiones}

Como se puede observar en el modelo desarrollado, existen ciertas capacidades personales e interpersonales que influyen en la competencia del liderazgo del administradoreducativo. Los factores críticos de las variables personales son: estar dispuesto a tomar una decisión difícil, tener confianza para tomar riesgos calculados, responsabilizarse por las actividades programadas y sus resultados, y perseverar cuando las cosas no están saliendo de acuerdo con lo planeado. En relación con las variables interpersonales los factores críticos son: entender cómo los diferentes grupos que conforman mi universidad operan y muestran su influencia en diferentes situaciones, trabajar constructivamente con personas que están en contra, o bien son muy entusiastas, desarrollar y utilizar redes de colegas para solucionar problemas clave, dar y recibir retroalimentación constructiva ya sea de colegas o de otras personas.

Estos comportamientos se relacionan con la variable de competencia del liderazgo en cuanto a: comprender el papel de la gestión de riesgos y los problemas legales de mi trabajo, entender cómo las universidades operan, ayudar a mi equipo a aprender cómo llevar a cabo los cambios necesarios con eficiencia, ser capaz de manejar mi propio proceso de aprendizaje y desarrollo profesional, ser capaz de manejar las tecnologías de información efectivamente para comunicar y desarrollar funciones de trabajo clave, ser capaz de hacer presentaciones efectivas para diferentes grupos de interés. Es por esto que es posible afirmar, con un coeficiente de determinación $\left(R^{2}\right)$ ajustado de $0.628(t=7.63, p \leq 0.001)$, que estadísticamente la hipótesis de trabajo se cumple a un nivel de significancia de 0.01 . La $\mathrm{R}^{2}$ indica el ajuste del modelo, por lo tanto, éste explica una buena parte de la variabilidad. El modelo presentado reafirma los estudios de Ramsden (1998) y de Splendlove (2007), en donde existen características personales e interpersonales que se presentan en el líder 
administrador universitario. Como se puede observar en los resultados los coeficientes de relación entre las variables

Las variables capacidades interpersonales y capacidades personales contribuyen a explicar de manera significativa la variable competencias del liderazgo como se explicó anteriormente. Sin embargo, la contribución de la variable capacidad interpersonal es mayor estadísticamente hablando que la de la variable capacidad personal en la explicación general del modelo. Es decir, de acuerdo a los resultados, es importante considerar en un administrador-educativo el fomento de las habilidades interpersonales y en un segundo plano las habilidades personales. El modelo resultante difiere del modelo propuesto por Scott et al. (2008) debido a que no todas las características personales e interpersonales contribuyen a explicar la competencia del liderazgo. Es preciso entender que el ámbito del estudio fueron universidades mexicanas y esto posiblemente haga una diferencia con respecto al modelo propuesto por estos autores para las universidades australianas.

Es muy importante mencionar que en el documento emitido por la Unesco (2000) se privilegia el trabajo en equipo y la integración horizontal de las organizaciones educativas. La recomendación para los directores de las IES de educación superior es que cuando se seleccione a alguna persona para desempeñar un puesto de jefatura en una IES, a cualquier nivel, se tomen en consideración las características interpersonales ya que son las que le ayudarán a cumplir las metas organizacionales como líder. Las habilidades personales también son importantes en cuanto a su impacto en el liderazgo y se debe buscar principalmente, la honestidad y la confianza en sí mismo como lo mencionan Ramsden (1998) y Spendlove (2007). Estos mismos criterios deben de ser considerados a la hora de buscar capacitación en el personal de este tipo dentro de las universidades.

Una variable importante de mencionar y que no fue incluida en este estudio es la funcionalidad organizacional de los sistemas educativos en cuanto a la estructura. En muchos casos, las estructuras de las universidades públicas no son eficientes y no corresponden a las necesidades del entorno, como lo menciona la Unesco (2000) en su documento "Desafíos de la Educación". En este sentido, es necesario realizar estudios que ayuden a entender los problemas organizacionales que se generan a raíz de las estructuras y que no facilitan que los líderes de la administración universitaria logren sus metas.

Otra línea de investigación que se desprende del presente estudio es el cómo profesionalizar la función del administrador-educativo en los diferentes niveles académicos. Es necesario que las autoridades educativas comprendan que la dimensión de esta posición 
es diferente a la de un docente, a la de un investigador o a la de un ejecutivo de una empresa. Como se expuso en este trabajo, existen diferencias con otro tipo de administradores y líderes, por lo que se requiere de una cuidadosa formación.

\section{Agradecimientos}

Agradecemos los fondos recibidos para la realización del proyecto a la Universidad de la Salle Bajío, México.

\section{Referencias}

Antonakis, John, Avolio, Bruce J. y Sivasubramaniam, Nagaraj. (2003). Context and leadership: An examination of the nine-factor full-range leadership theory using the Multifactor Leadership Questionnaire. The leadership quarterly, 14(3), 261-295. https://doi.org/10.1016/S1048-9843(03)00030-4

Bagozzi, Richard P. y Yi, Youjae. (1988). On the evaluation of structural equation models. Journal of the academy of marketing science, 16(1), 74-94.

Bass, Bernard. (1985). Leadership and performance beyond expectations. New York, USA: Free Press; Collier Macmillan.

Bass, Bernard. (1997). Does the transactional-transformational leadership paradigm transcend organizational and national boundaries? American psychologist, 52(2), 130.

Bass, Bernard y Stogdill, Ralph M. (1990). Handbook of leadership (Vol. 11). New York, USA: Free Press.

Becher, Tony y Trowler, Paul. (2001). Academic tribes and territories: Intellectual enquiry and the culture of disciplines. UK: McGraw-Hill Education.

Berdrow, Iris. (2010). King among Kings: Understanding the Role and Responsibilities of the Department Chair in Higher Education. Educational Management Administration \& Leadership, 38(4), 499-514.

Black, Simon A. (2015). Qualities of Effective Leadership in Higher Education. Open Journal of Leadership, 4(02), 54-66. doi: http://dx.doi.org/10.4236/ojl.2015.42006

Blake, Robert y Mouton, Jane. (1985). The Managerial Grid III: The Key to Leadership Excellence. Houston USA: Gulf Publishing Co.

Bryman, Alan. (2007). Effective leadership in higher education: A literature review. Studies in Higher Education, 32(6), 693-710. doi: http://dx.doi.org/10.1080/03075070701685114

Bush, Tony. (2007). Educational leadership and management: theory, policy, and practice. South African journal of education, 27(3), 391-406. 
Castaño, Nathalie, de Luque, Mary S., Wernsing, Tara, Ogliastri, Enrique, Shemueli, Rachel G., Fuchs, Rosa M. y Robles-Flores, José A. (2015). El Jefe: Differences in expected leadership behaviors across Latin American countries. Journal of World Business, 50(3), 584-597. doi: http://dx.doi.org/10.1016/j.jwb.2014.12.002

Chin, Wynne W. y Newsted, Peter R. (1999). Structural equation modelling analysis with small samples using partial least squares. En Rick H. Hoyle (Ed.), Statistical strategies for small sample research (pp. 307-341). Thousand Oaks, California, USA: Sage.

Cortina, José M. (1993). What is coefficient alpha? An examination of theory and applications. Journal of applied psychology, 78(1), 98-104.

Day, Donald V., Fleenor, John W., Atwater, Leanne E., Sturm, Rachel E. y McKee, Rob A. (2014). Advances in leader and leadership development: A review of 25years of research and theory. The Leadership Quarterly, 25(1), 63-82. doi: http://dx.doi.org/10.1016/i.leaqua.2013.11.004

De la Garza Aguilar, Javier. (2008). Evaluación y acreditación de la educación superior en América Latina y el Caribe. En Carlos Tünnermann (Ed.), La educación superior en América Latina y el Caribe: diez años después de la Conferencia Mundial de 1998 (pp. 175-222). Bogotá: Pontificia Universidad Javeriana and IESALC/Unesco.

De la Garza Carranza, María Teresa. (2011). Gestión en instituciones de educación superior. México: Gernika.

Dinh, Jessica E., Lord, Robert G., Gardner, William L., Meuser, Jeremy D., Liden, Robert C. y $\mathrm{Hu}$, Jinyu. (2014). Leadership theory and research in the new millennium: Current theoretical trends and changing perspectives. The Leadership Quarterly, 25(1), 36-62. doi: http://dx.doi.org/10.1016/j.leaqua.2013.11.005

Fiedler, Fred E., y Garcia Joseph E. (1987). New approaches to effective leadership: Cognitive resources and organizational performance. Oxford, England: John Wiley \& Sons.

Field, Andy. (2005). Discovering statistics with SPSS. London, England: Sage

Friedman, Jorge. (2003). Estrategias administrativas para la eficiencia universitaria. Estado, gobierno, gestión pública. Revista Chilena de Administración Pública, (6), 197-209.

Graen, George B. y Uhl-Bien, Mary. (1995). Development of leader-member exchange (LMX) theory of leadership over 25 years: Applying a multi-level multi-domain perspective. Leadership Quarterly, 6(2), 219-247.

Greenleaf, Robert K., y Spears, Larry C. (2002). Servant leadership: A journey into the nature of legitimate power and greatness. Paulist Press.

Goleman, David. (1998). Working with emotional intelligence. NY: Bantam Books 
Hair Jr, Joseph F., Hult, Thomas M., Ringle, Christian y Sarstedt, Marco. (2013). A primer on partial least squares structural equation modeling (PLS-SEM). Thousand Oaks, California: USA: Sage.

Hersey, Paul y Blanchard, Kenneth H. (1993). Management of organizational behavior: Utilizing human resources ( $6^{\text {th }}$ ed.). Englewood Cliffs, NJ: Prentice Hall.

Instituto Mexicano de la Juventud. (2012). Encuesta Nacional de valores en Juventud 2012. Recuperado de https://www.imjuventud.gob.mx/imgs/uploads/ENVAJ 2012.pdf

Jones, Sandra, Lefoe Geraldine, Harvey Marina y Ryland Kevin. (2012). Distributed leadership: a collaborative framework for academics, executives and professionals in higher education. Journal of Higher Education Policy and Management, 34(1), 67-78. doi: http://dx.doi.org/10.1080/1360080X.2012.642334

Judge, Timothy A., y Piccolo, Ronald F. (2004). Transformational and transactional leadership: a meta-analytic test of their relative validity. Journal of applied psychology, 89(5), 755.

Lévy-Mangin, Jean P. y Varela, Jesús. (2006). Modelización con estructuras de covarianzas en ciencias sociales. Coruña, España: Netbiblo.

López Yáñez, Julián, y Sánchez Moreno, María Rita. (2009). Mujeres agentes de cambio en la dirección de organizaciones universitarias. Revista de Educación, (348), 331-353.

McArthur, Ronald C. (2002). Democratic Leadership and Faculty Empowerment at the Community College: A Theoretical Model for the Department Chair. Community College Review, 30(3), 1-10.

Mumford, Michell D., Antes, Alison L., Caughron, Jay J. y Friedrich, Tamara L. (2008). Charismatic, ideological, and pragmatic leadership: Multi-level influences on emergence and performance. The Leadership Quarterly, 19(2), 144-160. https://doi.org/10.1016/j.leaqua.2008.01.002

Northouse, Peter G. (2013). Leadership: Theory and practice. United States: Sage publications.

Palacios Blanco, José L. y Vargas Chanes, Delfino. (2011). Medición efectiva de la calidad. México: Trillas.

Pounder, James S. (2001). "New leadership" and university organisational effectiveness: exploring the relationship. Leadership \& Organization Development Journal, 22(6), 281-290. doi: http://dx.doi.org/10.1108/EUM0000000005827

Ramsden, Paul. (1998). Managing the effective university. Higher education research \& development, 17(3), 347-370.

Ringle, Christina M., Wende, Sven, y Will, Alexander. (2005). SmartPLS 2.0. Recuperado de https://www.smartpls.com/faq/documentation/how-to-cite-smartpls 
Rossiter, John R. (2002). The C-OAR-SE procedure for scale development in marketing. International journal of research in marketing, 19(4), 305-335. doi: http://dx.doi.org/10.1016/S0167-8116(02)00097-6

Sánchez Gómez, Mirna, De la Garza Carranza, María Teresa y Cervantes Maldonado, Alfonso (octubre, 2013). Validación de un instrumento para la evaluación del perfil del administrador educativo de una institución de educación superior. En Congreso Internacional de Contaduría, Administración e Informática. 2-4 de octubre, Ciudad Universitaria, UNAM, México. Recuperado de http://congreso.investiga.fca.unam.mx/docs/xviii/docs/3.25.pdf

Sistema Nacional de Información Estadística Educativa. (2016a). Estadística e Indicadores Educativos por Entidad Federativa. Estadística del Sistema Educativo Guanajuato. Recuperado de http://www.snie.sep.gob.mx/descargas/estadistica e indicadores/estadistica e indica dores educativos 11GTO.pdf

Sistema Nacional de Información Estadística Educativa (2016b). Estadística e Indicadores Educativos por Entidad Federativa. Estadística del Sistema Educativo República Mexicana. Recuperado de http://www.snie.sep.gob.mx/descargas/estadistica e indicadores/estadistica e indica dores educativos 33Nacional.pdf

Scott, Geoff, Coates, Hamish y Anderson, Michelle. (2008). Learning leaders in times of change: Academic leadership capabilities. Australian higher education [versión digital en pdf]. Recuperado de http://works.bepress.com/hamish coates/3/

Spendlove, Marion. (2007). Competencies for effective leadership in higher education. International Journal of Educational Management, 21(5), 407-417.

Stogdill, Ralph. M. (1974). Handbook of leadership: A survey of theory and research. New York, USA: Free Press.

Unesco. (2000). Desafíos de la Educación. Recuperado de http://www.buenosaires.iipe.unesco.org/sites/default/files/modulo01.pdf

Valdéz Blanco, Dindo. (2010) Regresión por Mínimos Cuadrados Parciales. Revista Varianza, (7), 18-22. Recuperado de http://www.revistasbolivianas.org.bo/scielo.php?pid=S9876$\underline{67892010000100005 \& \text { script }=\text { sci arttext }}$

Wold, Herman. (1975). Path Models with Latent Variables. The NIPALS Approach. In Hubert M. Blalock (Ed.), Quantitative Sociology (pp. 307-357). New York: Seminar Press.

Wong, Ken K. (2013). Partial least squares structural equation modeling (PLS-SEM) techniques using SmartPLS. Marketing Bulletin, 24(1), 1-32. 